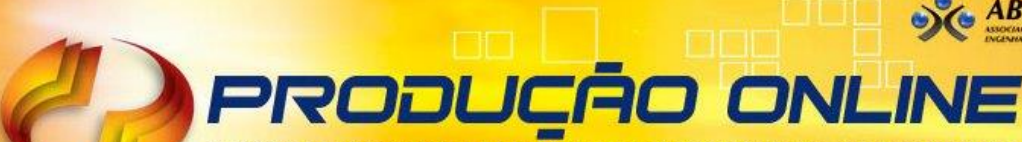 \\ REVISTA CIENTIFICA ELETRÓNICA DE ENGENHARIA DE PRODUÇÃO \\ ISSN 1676-1901
}

\section{APLICAÇÃO DE MÉTODOS ESTATÍSTICOS COM SUAVIZAÇÃO EXPONENCIAL DUPLA E TRIPLA PARA PREVISÃO DE DEMANDA NA GESTÃO DE ESTOQUES}

\section{APPLICATION OF STATISTICAL METHODS WITH EXPONENTIAL SMOOTHING DOUBLE AND TRIPLE FOR DEMAND FORECASTING IN THE INVENTORY MANAGEMENT}

\author{
Custodio da Cunha Alves* E-mail: custodio.alves@gmail.com \\ Edgar Hoepers* E-mail: edgarhoepers@hotmail.com \\ Emerson Jose Corazza* E-mail: emersoncorazza@univille.br \\ Gilson João dos Santos* E-mail: gilson.joao@univille.br \\ Renato Cristofolini* E-mail: renato.cristofolini@univille.br \\ Altair Carlos da Cruz* E-mail: altaircruz@yahoo.com.br \\ *Centro de Aplicação Mecânica e Gestão Industrial (CAMEGI), Universidade da \\ Região de Joinville (UNIVILLE), Joinville, SC
}

Resumo: Os métodos estatísticos com suavização exponencial dupla e tripla amplamente utilizados para modelar tendências significativas em dados não estacionários por séries temporais são aplicados neste trabalho para obter em curto prazo, previsões para o planejamento da demanda do processo produtivo em uma indústria metalúrgica do norte de Santa Catarina. O objetivo principal da aplicação de tais métodos é estabelecer a previsão de demanda com o propósito de antecipar cenários futuros de vendas em duas categorias de produtos e obter o melhor aproveitamento da capacidade produtiva através de uma gestão de estoques adequada para a redução de riscos no processo de tomada de decisões gerenciais dessa indústria. Os resultados obtidos com a seleção apropriada dos métodos preditivos com suavização exponencial, objeto de estudo deste trabalho, foram fundamentais para o analista do sistema de previsão de demanda direcionar especial atenção ao grau de acurácia que integrado a uma política de gerenciamento de estoques eficiente foi responsável por minimizar os efeitos de variabilidade e custos operacionais, além contribuir na melhoria dos níveis de serviço e no consequente aumento da rentabilidade da indústria envolvida.

Palavras-chave: Métodos estatísticos. Suavização exponencial. Previsão. Gestão de estoques.

\begin{abstract}
The statistical methods with double and triple exponential smoothing widely used to model significant trends in non-stationary time series data are applied in this work to obtain short-term forecasts for the planning of the demand of the productive process in a metallurgical industry of the north of Santa Catarina. The main objective of the application of such methods is to establish demand forecasting in order to anticipate future sales scenarios in two categories of products and obtain the best utilization of productive capacity through adequate inventory management to reduce risks in the process decisionmaking in this industry. The results obtained with the appropriate selection of the predictive methods with exponential smoothing, object of study of this work, were fundamental for the analyst of the system of forecast of demand to direct special attention to the degree of accuracy that integrated to an efficient inventory management policy was responsible per minimizing the effects of variability and operational costs, as well as contribute to the improvement of service levels and the consequent increase in the profitability of the industry involved.
\end{abstract}

Keywords: Statistical methods. Exponential smoothing. Forecasting. Inventory management. 


\section{INTRODUÇÃO}

Os métodos com suavização exponencial dupla e tripla são técnicas de previsão apropriadas para dados não estacionários de séries temporais nos quais existem tendências significativas no decorrer do tempo. Nesses métodos, a tendência reflete a nítida influência de fatores de longo prazo que afetam a série temporal de modo bem coerente e gradual no decorrer do tempo, ou seja, reflete mudanças nos dados que ocorrem ao longo do tempo. Entretanto, não é raro os dados de séries temporais exibirem algum tipo de tendência para cima ou para baixo no decorrer do tempo (RAGSDALE, 2017).

Este trabalho propõe a aplicação desses métodos com suavização exponencial para estabelecer em curto prazo, previsões de séries temporais com modelagem em ambiente MS-Exce|® para o planejamento de demanda de duas categorias de produtos produzidos numa empresa metalúrgica localizada na região norte do estado de Santa Catarina. O objetivo deste trabalho é estabelecer a previsão de demanda para obter o melhor aproveitamento da capacidade produtiva através de uma gestão de estoques adequada, aplicando métodos estatísticos com suavização exponencial dupla e tripla para antecipar cenários futuros de vendas em tais categorias de produtos, tendo como propósito a redução de riscos na tomada de decisão dessa empresa.

A estrutura do método com suavização exponencial dupla de Holt proposto neste trabalho cuja série histórica de vendas do produto requer a definição de dois coeficientes de suavização, um para cada componente sistemático da demanda (nível e tendência, $\alpha$ e $\beta$ ) e o método com suavização tripla de Holt-Winter cuja série histórica de vendas do produto requer a definição três parâmetros estatísticos de ajuste (constantes ou coeficientes de suavização), em cada componente sistemático da demanda nível ( $\alpha$ ), tendência ( $\beta$ ) e sazonalidade $(\gamma)$.

O artigo está estruturado em cinco seções, incluindo a presente introdução. A seção 2 apresenta o referencial teórico sobre métodos estatísticos com suavização exponencial envolvendo a estrutura dos métodos com suavização exponencial dupla e tripla bem como complementos básicos fundamentais que auxiliam nas interpretações e análise inerentes a estas técnicas de previsão; na seção 3 , é apresentado a metodologia utilizada cuja sistemática de aplicação consiste em 
selecionar o método estatístico com suavização exponencial apropriado para obter a melhor previsão de demanda. Na sequência, os resultados são apresentados e discutidos; e finalmente, a seção 4, apresenta as considerações finais.

\section{REFERENCIAL TEÓRICO}

Esta seção traz inicialmente alguns fundamentos básicos, tais como à análise de séries temporais, preliminares ao estudo de métodos estatísticos de previsão. Em seguida, são apresentados os principais métodos estatísticos com suavização exponencial para realizar prognósticos de variáveis atribuindo maior ênfase aos métodos de previsão apropriados a dados não estacionários em particular, os métodos de suavização exponencial dupla cujas séries temporais exibem uma tendência linear e tripla que exibem tanto tendência quanto sazonalidade. Estes métodos são amplamente aplicados para estabelecer previsão de curto prazo da demanda, objeto de estudo deste trabalho.

Além disso, são concentrados nesse referencial teórico estudos complementares ao entendimento e a aplicação dos métodos estatísticos de previsão por séries temporais que auxiliam nas interpretações e análises inerentes as estas técnicas de previsão tais como os princípios de avaliação e monitoramento de desempenho de métodos de previsão e o estudo da previsão de demanda integrada a gestão de estoques, atividades principais de apoio à logística ligadas à posse e movimentação dos produtos nas organizações.

\subsection{Métodos Estatísticos de Previsão por Séries Temporais}

A aplicação de métodos estatísticos de previsão por séries temporais apropriados para prever quantitativamente uma variável e medir a qualidade da previsão estabelecida tem sido cada vez mais utilizada uma vez que representa um diferencial para antecipar cenários futuros (VERÍSSIMO et al., 2012; JUÁREZ, et al., 2016; GARCÍA-DÍAZ, 2016). Neste contexto, o objetivo da análise de séries temporais é encontrar modelos (estatísticos e/ou matemáticos) que permitem descrever de forma adequada o processo de geração de dados capaz de prever o comportamento futuro dessa variável para um determinado horizonte de planejamento. 
A essência dos métodos de previsão que utilizam análise de séries temporais consiste em identificar o padrão da série, separando-o do ruído contido nas observações individuais, e utilizá-lo para prever os valores futuros da série. Segundo Morettin e Toloi (2006) esses métodos aplicados para descrever séries temporais são considerados processos estocásticos, ou seja, processos cuja evolução no tempo é gerada e controlada por leis probabilísticas.

Uma vez que muitos métodos estatísticos de previsão estão à disposição para a modelagem de dados por séries temporais, cada qual com suas capacidades e limitações, é impossível saber de antemão qual método será mais eficiente para determinado conjunto de dados. Assim, a seleção do método estatístico de previsão por série temporal mais eficiente depende de vários fatores, tais como o comportamento do fenômeno observável ou o conhecimento a priori, que se tenha sobre a sua natureza e do objetivo da análise.

As duas principais famílias de modelos matemáticos utilizadas no estudo de previsão por séries temporais: os modelos de Box-Jenkins partem da ideia de que os valores de uma série temporal são altamente dependentes, ou seja, cada valor pode ser explicado por valores prévios da série e os modelos de Suavização Exponencial, objeto de estudo desse trabalho, utilizam uma ponderação distinta para cada valor observado na série temporal, de modo que valores mais recentes recebam pesos maiores.

\subsection{Métodos Estatísticos de Suavização Exponencial}

Os métodos estatísticos com suavização exponencial são amplamente utilizados para fornecer previsões de curto prazo para os dados de vendas e níveis de demanda, devido a sua simplicidade, baixo custo de operação, boa precisão, capacidade de ajustamento automático e rápido a mudanças na série temporal em análise. Além disso, são potencialmente mais vantajosos do que outros métodos de previsão estatística, pois não exigem que haja um número mínimo de observações a serem realizadas antes da previsão (YORK, 2015).

O procedimento de um método estatístico de previsão com suavização ou ajuste exponencial parte de uma equação de médias móveis, ponderadas 
exponencialmente, com o objetivo de produzir ajustes nas variações aleatórias dos dados de determinada série temporal (RAGSDALE, 2017).

Os métodos de previsão com suavização exponencial se utilizam apenas de observações da própria série de dados, não dependendo de nenhuma variável externa para realizar previsões (SOUZA, SAMOHYL \& MIRANDA, 2008). São reconhecidos como uma das ferramentas mais empregadas na previsão que envolve curto prazo de séries temporais e a popularidade desses métodos se deve principalmente à sua simples formulação, facilidade de ajustes e boa precisão (GARDNER, 1985, 2006; BERMÚDEZ, SEGURA \& VERCHER, 2008).

Os procedimentos de um método com suavização exponencial incorporam de maneira simples e compreensível o nível, a tendência e a sazonalidade que apresentam os dados da série temporal, assim como o erro de previsão, tanto na forma aditiva quanto na forma multiplicativa que é o componente estocástico do método. A seguir são apresentadas as três principais variações básicas com suavização exponencial: simples, dupla e tripla, comumente utilizadas na prática.

\subsubsection{Método Estatístico com Suavização Exponencial Simples}

O tipo mais simples de série temporal é aquele em que os valores da série flutuam aleatoriamente em torno de um valor fixo, sem apresentar qualquer tendência. Se a série temporal se mantém constante sobre um nível médio, uma suavização exponencial simples proposta por Brown (1959) pode ser utilizada para a previsão de valores futuros da série.

O método estatístico com suavização exponencial simples para a previsão por séries temporais é apropriado para dados estacionários nos quais não há tendência significativa nos dados no decorrer do tempo.

Os modelos exponenciais com ajuste assumem a representação matemática dada por (MAKRIDAKIS, WHEELWRIGHT\& HYNDMAN, 2008)

$$
\hat{Y}_{t+1}=\hat{Y}_{t}+\alpha\left(Y_{t}-\hat{Y}_{t}\right) \quad 0 \leq \alpha \leq 1
$$

como parte de uma equação de médias móveis, ponderadas exponencialmente, com 
o objetivo de produzir ajustes nas variações aleatórias dos dados de determinada série temporal. Este procedimento denominado suavização exponencial simples utiliza uma ponderação distinta para cada valor observado na série temporal, de modo que valores mais recentes recebam pesos maiores. Dessa forma, os pesos formam um conjunto que decai exponencialmente a partir de valores mais recentes (HYNDMAN \& ATHANASOPOULOS, 2018).

A equação (1) indica que o valor previsto para o período de tempo $t+1\left(\hat{Y}_{t+1}\right)$ é igual ao valor previsto $\left(\hat{Y}_{t}\right)$ mais um ajuste para o erro originado na previsão do valor no período anterior $\alpha\left(Y_{t}-\hat{Y}_{t}\right)$. O parâmetro $\alpha$ denominado constante de suavização do método; que pode assumir qualquer valor entre 0 e $1(0 \leq \alpha \leq 1) ; Y_{t}$ é o valor observado atual (valor real) para o período de tempo $t$ e, $\hat{Y}_{t}$ é o valor previsto para o mesmo período de tempo t. Assim, o valor previsto para o próximo período $\hat{Y}_{t+1}$ é uma combinação do valor previsto atual $\hat{Y}_{t}$ e o valor observado atual $Y_{t}$. O valor da constante de suavização $\alpha$ é arbitrário; a determinação de seu melhor valor pode ser realizada iterativamente, utilizando alguma forma de comparação, como, por exemplo, o erro quadrático médio (MSE). Desta forma, seleciona-se aleatoriamente um valor inicial para a constante, a partir do qual previsões são geradas. Comparam-se os valores previstos com os valores reais, e calcula-se a média do quadrado das diferenças entre os mesmos; o parâmetro que minimiza essa média é utilizado no modelo final (PELLEGRINI \& FOGLIATTO, 2001).

Nas próximas seções, são apresentados os métodos estatísticos de previsão que são apropriados para séries temporais não estacionárias envolvendo uma tendência significativa nos dados no decorrer do tempo.

\subsubsection{Método com Suavização Exponencial Dupla (Método de Holt)}

O método com suavização exponencial dupla (também conhecido como método de Holt) é em geral, uma ferramenta eficaz de previsão para dados por série temporal que exibem uma tendência linear. Desenvolvida por Holt (1957) que ampliou a suavização exponencial simples para dados de séries temporais que apresentam 
tendência linear. Este método oferece refinamentos adicionais na modelagem, à medida que introduz uma constante de suavização que afeta a tendência da série. A função de previsão do método de Holt é representada por

$$
\hat{Y}_{t+n}=E_{t}+n T_{t}
$$

onde a previsão para o período de tempo $t+n\left(\hat{Y}_{t+n}\right)$ é igual a estimativa do nível esperado da série temporal no período de tempo $t\left(Y_{t}\right)$ mais a influência esperada da tendência (taxa de aumento ou redução) durante os próximos $\mathrm{n}$ períodos $(n T)$. A função de previsão na equação (2) pode ser utilizada para obter períodos de tempo n para previsões no futuro, onde $n=1,2,3$, e assim por diante.

O método desenvolvido por Holt recorre à utilização dos parâmetros $\alpha$ e $\beta$ denominadas constantes de suavização. Nesse método, além da equação (2) que calcula a previsão, duas outras funções são utilizadas para estimar o nível e a tendência da série temporal, conforme equações (3) e (4), respectivamente.

$$
\begin{array}{ll}
E_{t}=\alpha Y_{t}+(1-\alpha)\left(E_{t-1}+T_{t-1}\right), & 0 \leq \alpha \leq 1 \\
T_{t}=\beta\left(E_{t}-E_{t-1}\right)+(1-\beta) T_{t-1}, & 0 \leq \beta \leq 1
\end{array}
$$

onde $E_{t}$ é a estimação para o nível da série no período t e $T_{t}$ é a estimação da inclinação (tendência) da série no mesmo período $t$. A equação (2) ajusta diretamente $E_{t}$ para a tendência do período anterior $T_{t-1}$, adicionando o último valor suavizado $E_{t-1}$

Os valores de $\alpha$ e $\beta$ representa as constantes (parâmetros ) de suavização que são encontrados por ensaio sobre a série histórica, sendo frequente selecionar aqueles valores que minimizam o erro quadrático médio. Para enfrentar o problema da subjetividade na seleção dos parâmetros $\alpha$ e $\beta$ algumas investigações desenvolvidas (BILLAH; SNYDER; KOEHLE, 2006; GELPER; FRIED; CROUX, 2010) mostram como selecionar esses parâmetros otimizando algumas das medidas de desempenho dos prognósticos, como erro médio (ME), erro absoluto médio (MAE) e erro quadrático médio (MSE). Enquanto isso, Rasmussen (2004), propõe o cálculo tanto dos parâmetros $\alpha$ e $\beta$ que otimizam o método de previsão quanto dos valores 
iniciais, definindo para tal o MSE como função objetivo para minimizar e resolver o método, com auxílio da ferramenta solver no ambiente MS-Excel®.

\subsubsection{Método com Suavização Exponencial Tripla (Método de Holt-Winter)}

Os métodos estatísticos por séries temporais são técnicas quantitativas frequentemente utilizadas para realizar prognósticos de variáveis, dentre os quais se encontram os métodos com suavização exponencial, em particular o método de suavização exponencial tripla (também conhecido como método de Holt-Winter) que é uma expansão do método de previsão de Holt (1957). Desenvolvido por Winter (1960) para aplicar à série temporal que exibe tendência e sazonalidade, é um dos métodos mais utilizados para previsão de curto prazo da demanda, devido a sua simplicidade, baixo custo de operação, boa precisão, capacidade de ajustamento automático e rápido a mudanças na série em análise. Partindo do princípio que determinadas séries possuem um fator sazonal, além do nível e tendência que capta características da série que se repetem a intervalos regulares de tempo, Winter propõe métodos de projeção para essas séries, considerando dois tipos de efeitos sazonais: aditivos e multiplicativos.

a) Método de Holt-Winter para Efeitos Sazonais Aditivos

O método de Holt-Winter para efeitos sazonais aditivos é utilizado na modelagem de dados onde á amplitude do ciclo sazonal independe do nível local da série, ou seja, permanece constante com o passar do tempo.

Seja um modelo cuja série sazonal, de período $\mathrm{p}$, é formada pela soma de: nível, tendência, um fator sazonal e um erro aleatório, ou seja, o modelo é dado por

$$
Y_{t}=E_{t}+T_{t}+S_{t}+\epsilon_{t} \quad t=1,2, \ldots
$$

As projeções dos valores futuros da série são efetuadas através da função de previsão do método representada por 


$$
\hat{Y}_{t+n}=E_{t}+n T_{t}+S_{t+n-p}, \mathrm{t}=1,2 \ldots
$$

onde $\hat{Z}_{t+n}$ é a previsão para $n$ períodos à frente $(t+n)$. Nesse método, além da função (6) que calcula a previsão, três outras funções são utilizadas para estimar o nível, a tendência da série no período atual e os valores do fator sazonal correspondente ao último período de sazonalidade, conforme equações (7), (8) e (9), respectivamente.

$$
\begin{array}{ll}
E_{t}=\alpha\left(Z_{t}-S_{t-p}\right)+(1-\alpha)\left(E_{t-1}+T_{t-1}\right), & 0<\alpha<1 \\
T_{t}=\beta\left(E_{t}-E_{t-1}\right)+(1-\beta) T_{t-1}, & 0<\beta<1 \\
S_{t}=\gamma\left(Y_{t}-E_{t}\right)+(1-\gamma) S_{t-p}, & 0<\gamma<1
\end{array}
$$

onde $\alpha, \beta$ e $\gamma$ são as constantes (parâmetros ) de suavização que controlam o peso relativo ao nível $\left(E_{t}\right)$, a tendência $\left(T_{t}\right)$ e a sazonalidade $\left(S_{t}\right)$, respectivamente. As equações (7) e (9) assumem que, no período de tempo t, existe uma estimativa do índice sazonal no período de tempo t-p ou que existe um valor $S_{t-p}$.

b) Método de Holt-Winter para Efeitos Sazonais Multiplicativos

Uma leve modificação no modelo anterior torna-se apropriado para a modelagem de dados de série temporal estacionária com efeitos sazonais multiplicativos. O método de Holt-Winter para efeitos sazonais multiplicativos é utilizado na modelagem de dados onde a amplitude do ciclo sazonal varia proporcionalmente ao nível da série com o passar do tempo.

Seja um modelo de série sazonal, de período $\mathrm{s}$, fator sazonal multiplicativo e tendência aditiva, isto é,

$$
Y_{t}=E_{t} S_{t}+T_{t}+\epsilon_{t}, t=1,2, \ldots
$$

descreve o comportamento estrutural da série, as projeções dos valores futuros da série são efetuadas através da função de previsão do método representada por

$$
\hat{Y}_{t+n}=\left(E_{t}+n T_{t}\right) S_{t+n-p} \quad n=1,2, \ldots
$$


onde $\hat{Y}_{t+n}$ é a previsão para n períodos à frente $(t+n)$. Nesse método, além da função (11) que calcula a previsão três outras funções são utilizadas para estimar o nível, a tendência da série no período atual e os valores do fator sazonal correspondente ao último período de sazonalidade, conforme equações (12), (13) e (14), respectivamente.

$$
\begin{array}{ll}
E_{t}=\alpha \frac{Y_{t}}{S_{t-p}}+(1-\alpha)\left(E_{t-1}+T_{t-1}\right), & 0 \leq \alpha \leq 1 \\
T_{t}=\beta\left(E_{t}-E_{t-1}\right)+(1-\beta) T_{t-1}, & 0 \leq \beta \leq 1 \\
S_{t}=\gamma \frac{Y_{t}}{E_{t}}+(1-\gamma) S_{t-p}, & 0 \leq \gamma \leq 1
\end{array}
$$

onde $\alpha, \beta$ e $\gamma$ são as constantes (parâmetros) de suavização que controlam 0 peso relativo ao nível $\left(E_{t}\right)$, a tendência $\left(T_{t}\right)$ e a sazonalidade $\left(S_{t}\right)$, respectivamente. As equações (12) e (14) assumem que, no período de tempo t, existe uma estimativa do índice sazonal no período de tempo $t-p$ ou que existe um valor $S_{t-p}$.

\subsection{Avaliação e monitoramento do desempenho de métodos de previsão}

A pesquisa por métodos estatísticos de previsão apropriados para prever quantitativamente uma variável e medir a qualidade de determinada previsão tem sido um diferencial cada vez mais utilizado pelas empresas para antecipar cenários futuros que contribuem para planejar, alocar e dimensionar recursos de modo a tentar reduzir custos desnecessários decorrentes de decisões equivocadas.

O processo para estabelecer previsões constitui-se em uma das mais importantes ações no que se refere a diversas tomadas de decisões no dia a dia de uma organização. Este processo compreende uma série de atividades relacionadas que transformam uma ou mais entradas em uma ou mais saídas. Todas as atividades de trabalho são realizadas em processos, e a previsão não é exceção (MONTGOMERY; JENNINGS; KULAHCI, 2016). A figura 1 ilustra estas atividades. 
Figura 1 - Processo de Previsão

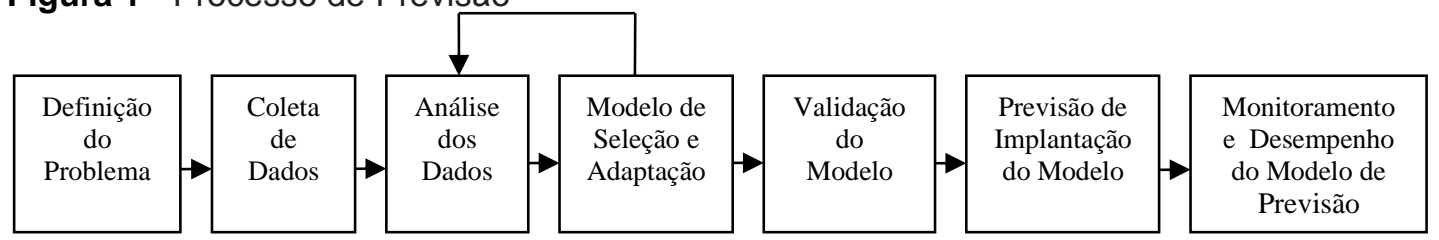

Fonte: Adaptado com base em Montgomery, Jennings \& Kulahci (2016, p.12)

O monitoramento e desempenho de modelo de previsão, última atividade de trabalho de um sistema de previsão conforme figura 1, deve ser uma atividade contínua após a implantação do método estatístico de previsão para garantir que ele ainda apresenta desempenho satisfatório.

Em previsão, é natural que determinadas condições mudem ao longo do tempo, o que implica que um método de previsão com bom desempenho no passado, com o passar do tempo, tenha seu desempenho alterado. Normalmente, a redução do desempenho de um método, resulta em erros maiores ou mais sistemáticos de previsão. Consequentemente, o monitoramento dos erros de previsão se constitui em uma parte essencial do projeto de um bom sistema de previsão (CRUZ, et al.,2014).

Para analisar a forma de avaliar o desempenho de um modelo de previsão para uma série temporal em particular ou aplicação, é importante definir com cuidado o significado de desempenho. A avaliação do desempenho, com base no ajuste da previsão ou modelo por séries temporais de dados históricos é considerada atraente em qualquer sistema de previsão. Há muitas medidas estatísticas que descrevem a forma como um modelo se encaixa em uma determinada amostra de dados, e algumas delas são descritas nesta seção.

O usuário de previsão, muitas vezes está muito preocupado com a precisão da previsão futura, e não com o modelo de qualidade do ajuste, por isso é importante avaliar esse aspecto em qualquer modelo de previsão recomendado.

A medida quantitativa de acurácia ou precisão da previsão deve sempre ser avaliada como parte de um esforço de validação do modelo. Assim, validar o desempenho de um ou mais modelos a partir do desenvolvimento de um modelo de previsão para os resíduos envolve provavelmente algum tipo de amostra dividida ou procedimento de validação cruzada. Quando mais de um modelo de previsão parecer razoável para uma determinada aplicação, essas medidas de precisão do tempo também podem ser utilizadas para estabelecer diferenciação entre modelos 
concorrentes (CRUZ, et al.,2014). Costuma-se avaliar o desempenho do modelo de previsão usando os erros de previsão um passo a frente

$$
e_{t}(1)=y_{t}-\hat{y}_{t}(t-1)
$$

onde, $\hat{y}_{t}(t-1)$ é a previsão de $y_{t}$ estabelecida em um período anterior.

As medidas quantitativas mais formais de desempenho e otimização são em muitos casos de previsão, o principal critério de seleção de um modelo de previsão (LÓPEZ, MENDOZA \& MASINI, 2013). Essas medidas permitem a medição da eficácia de um modelo de previsão, mostrando o quanto ele é capaz de reproduzir os dados que já são conhecidos. Para o analista de previsão o diferencial para seleção de um método previsão está no grau de acurácia da previsão futura, ou seja, o método capaz de gerar previsões que sejam tão precisas quanto possível.

Dentre essas medidas estatísticas de desempenho e otimização que quantificam a acurácia ou grau de previsão, geralmente as mais utilizadas são apresentadas a seguir.

Suponha que existem $n$ observações para as quais as previsões foram estabelecidas e $n$ erros de previsão um passo à frente, $e_{t}(1), t=1,2, \ldots ., n$. As medidas padrão de precisão da previsão são o erro médio

$$
M E=\frac{1}{n} \sum_{t=1}^{n} e_{t}(1)
$$

o desvio médio absoluto (ou erro médio absoluto)

$$
M A E=\frac{1}{n} \sum_{t=1}^{n}\left|e_{t}(1)\right|
$$

e o erro quadrático médio

$$
M S E=\frac{1}{n} \sum_{t=1}^{n}\left[e_{t}(1)\right]^{2}
$$


O erro quadrático médio ( $M S E$ ) é considerado geralmente como a medida estatística mais utilizada por apresentar a vantagem de ser mais facilmente manipulável matematicamente.

\subsection{Previsão de Demanda e Gestão de Estoques}

A competitividade e o aumento da instabilidade do mercado global exigem que o tempo de tomada de decisão das empresas seja reduzido constantemente. A previsão de demanda é uma atividade importante, pois ela pode revelar as tendências de mercado e contribuir para o planejamento estratégico de uma empresa (BERMÚDEZ; SEGURA; VERCHER, 2008).

A previsão consiste na estimação e análise da demanda futura para um produto em particular, componente ou serviço, utilizando entradas como relações históricas de vendas, estimação de marketing e informação promocional através de diferentes técnicas de previsão. Neste sentido, a previsão como ferramenta logística inclui a previsão de demanda com o objetivo de melhorar o fluxo de informação na cadeia de abastecimento das empresas e, portanto, preparar a organização para efeitos técnicos, humanos e financeiros capazes de suportar operações futuras tais como: estimar compras, produção, necessidades de armazenagem, transportes, etc. (KRAJEWSKI, MALHOTRA \& RITZMAN, 2015).

A previsão de demanda é utilizada quando há necessidade de prever um parâmetro numérico para o qual os resultados passados são bons indicadores de comportamento futuro (SINGH, BAHL \& KUMAR, 2017). Além disso, constitui-se em uma das atividades de gestão mais importantes para a tomada de decisões gerenciais uma vez que o sucesso futuro de qualquer empresa depende muito de como o gestor é mais experiente em detectar tendências e desenvolvimento de estratégias adequadas (KRAJEWSKI, MALHOTRA \& RITZMAN, 2015). Neste contexto, a formulação de planejamentos e o direcionamento estratégico das empresas dependem da identificação e a previsão correta das mudanças emergentes no ambiente de negócios.

O fornecimento de previsões ou projeções de demanda se constitui atualmente, numa parte fundamental da logística pelas implicações que uma mudança na estratégia decorrente dos principais processos da cadeia de abastecimento (gestão 
de estoque, abastecimento, transporte, fabricação, nível de serviço, etc.) e para os benefícios que proporcionam sua correta estimativa e monitoramento (VERÍSSIMO et al., 2012).

A logística em muitas situações é uma ferramenta que aumenta a vantagem competitiva e reduz custos pois ela é hoje amplamente reconhecida como um centro de agregação de valor nas organizações através da disponibilidade de produtos, consistência de entregas, precisão no gerenciamento de estoque e demanda e facilidade de efetivar pedidos (COELHO, 2012; COELHO \& LAPORTE, 2013).

O gerenciamento adequado de estoques é uma forma de reduzir custos e atender ao mercado de maneira eficaz. Uma boa maneira de fazê-lo é utilizando a previsão de demanda. Esta é uma importante ferramenta de apoio para a tomada de decisões gerenciais (COUTO \& ESPÍRITO SANTO, 2013). Conforme, Vargas, Baccin \& Sellitto (2017), tal ferramenta pode auxiliar a minimizar os efeitos de variabilidade e custos operacionais, assim como melhorar os níveis de serviço e a rentabilidade das empresas.

A previsão de demanda é uma variável muito importante para a gestão de estoques. E essa gestão é a principal preocupação ao se realizar a análise mercadológica de preferência por um ou outro produto.

Segundo Coelho (2017) todos os principais problemas na gestão de estoques decorre de falhas na gestão e previsão da demanda no processo de tomada de decisão. Além disso, o correto dimensionamento de estoques é essencial para a conservação da competitividade empresarial no cenário em que o mercado se encontra atualmente. Tanto para evitar rupturas quanto para impedir que uma grande quantidade de produtos seja mantida em estoques, justifica-se a necessidade de antever a demanda, preparando-se com volumes adequados de estoques, uma vez que estas situações podem incorrer em elevados custos de operação (COELHO, 2008).

O estoque é um dos ativos mais caros de muitas empresas e representam uma boa parcela do capital total investido. Com a globalização, os gerentes de operações em todo o mundo há muito reconhecem que o bom gerenciamento de estoque é fundamental. Por um lado, uma empresa pode reduzir custos reduzindo estoques. Por outro, a produção pode parar e os clientes ficam insatisfeitos quando um item está em falta. O objetivo do gerenciamento de estoques é encontrar um equilíbrio entre o 
investimento em estoque e o atendimento ao cliente pois não se pode alcançar uma estratégia de baixo custo sem um bom gerenciamento de estoque (HEIZER, RENDER \& MUNSON, 2017). Segundo, Bowersox, et al. (2014), gerir estoque economicamente consiste na procura do equilíbrio entre demanda e consumo.

O valor do gerenciamento de estoques torna-se aparente quando a complexidade da cadeia de suprimentos é reconhecida. Uma questão fundamental no gerenciamento da cadeia de suprimentos é quanto ter em estoque. A resposta a essa pergunta envolve uma troca entre as vantagens e desvantagens de manter o estoque. Dependendo da situação, as pressões por ter estoques pequenos podem ou não exceder as pressões por ter grandes estoques (KRAJEWSKI, MALHOTRA \& RITZMAN, 2015).

A gestão de estoques faz parte das atividades principais de qualquer empresa, portanto, de sua adequada gestão dependerá os resultados das atividades subsequentes. É considerada como uma das alternativas econômicas mais utilizadas para reduzir a estrutura de custos dentro de uma organização. Isso é realizado através da análise da demanda, tipo de produto, condições de mercado, com base em modelos de inventários que permitem ajustar tal comportamento ao planejamento estratégico. Os modelos de estoques fundamentam-se no pressuposto de que os produtos têm uma vida útil limitada, no entanto, em alguns sistemas, sua deterioração é gerada mais rapidamente, resultando em custos mais altos. Além disso, os modelos de estoques fundamentam-se no pressuposto de que os produtos também possuem um tempo de vida ilimitado, entretanto, em alguns sistemas há uma geração de custos adicionais, associados à deterioração da qualidade dos produtos, denominados itens perecíveis (PINEDA; TORRES, 2018).

A modelagem de estoque trata da definição do nível de certa mercadoria que uma empresa deve manter para garantir uma operação tranquila. A base para a decisão é um modelo determinístico de estoque que equilibra o custo de capital resultante da permanência de excedente de estoque com o custo de multas resultante da falta de estoque. O principal fator que afeta a solução é a natureza da demanda: determinística ou estocástica.

Os modelos de inventário de itens perecíveis são classificados de acordo com o comportamento da demanda no sistema ou sua vida útil. De acordo com o tipo de demanda, se é determinística, a mudança de inventário pode ser determinada por uma 
previsão de demanda constante. No entanto, se é estocástica, pode ser representada através de uma função de probabilidade (PINEDA \& TORRES, 2018).

Neste trabalho a aplicação métodos estatísticos de séries temporais com suavização exponencial dupla e tripla é proposta para estabelecer a previsão de demanda com o propósito de antecipar cenários futuros de vendas em duas categorias de produtos e obter o melhor aproveitamento da capacidade produtiva através de uma gestão de estoques adequada para a redução de riscos na tomada de decisões gerenciais da empresa envolvida.

\section{METODOLOGIA DE APLICAÇÃO PARA A PREVISÃO DE DEMANDA}

A aplicação proposta neste trabalho consiste na utilização de modelos estatísticos com suavização exponencial dupla e tripla através de planilhas para determinar as previsões de demanda em um horizonte de quatro trimestres de 2016 . Para tal aplicação são utilizados dados reais referentes ao histórico de vendas de uma indústria metalúrgica do norte de Santa Catarina, cujos dados revelam tanto características de tendência quanto de tendência e sazonalidade simultaneamente.

As séries temporais referentes às vendas trimestrais de duas categorias de produtos dessa indústria com representação em faturamento e impactantes no processo produtivo que são os produtos eletroduto e bucha, identificados no contexto real durante o período de janeiro de 2011 até dezembro de 2015, totalizando 20 trimestres. Esses produtos tanto o eletroduto quanto a bucha são aplicados em instalações elétricas residenciais, comerciais e industriais. O eletroduto é desenvolvido em PVC e obtido através da extrusão do material em matriz. Sua função é a condução do cabeamento elétrico e de comunicação. A bucha é desenvolvida através da injeção do alumínio e usinagem de rosca. Sua função é a fixação de eletrodutos em caixa de derivação.

De posse, do histórico de vendas trimestrais das duas linhas de produtos estudados, informações estas obtidas através do relatório de gerenciamento do faturamento dessa empresa, a próxima etapa é a classificação dos dados para o estudo das séries temporais envolvidas nessa aplicação. A análise de séries temporais utilizada nesse trabalho envolve as demandas desses dois produtos eletroduto e bucha, distribuídos em duas linhas de produção. Durante esses cinco 
anos de operação essa indústria obteve um determinado crescimento na venda desses produtos conforme os gráficos da figura 2.

Figura 2 - Dados históricos de vendas dos produtos (em milhares) para a previsão de demanda
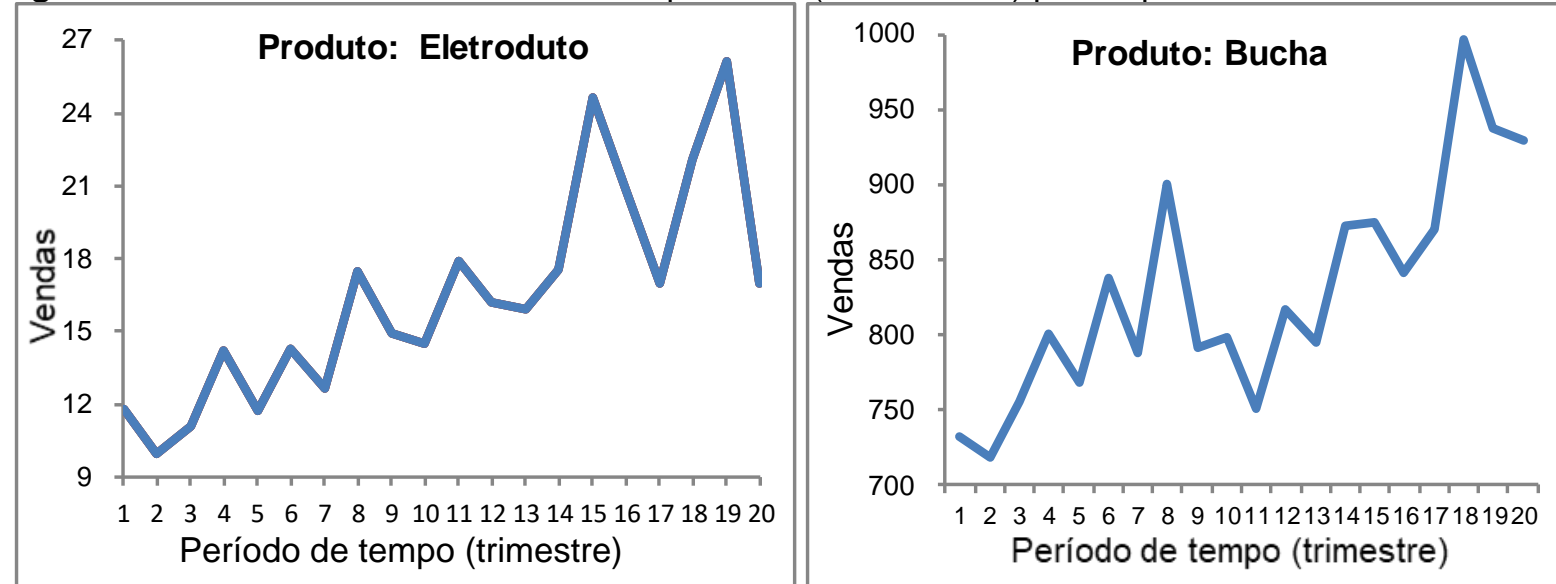

Fonte: Autores (2019)

Observa-se que ambas as séries temporais não estacionárias ilustram uma determinada tendência crescente nas vendas ao longo desse período. Esta análise indica uma possível aplicação de métodos com suavização exponencial que apresentem tais características.

Além da análise gráfica para prever o valor dessa variável de série temporal, realizou-se um estudo das medidas de desempenho e otimização dos métodos de previsão como, por exemplo, o MSE e as constantes de suavização exponencial que minimizam MSE para a previsão via método estatístico com suavização exponencial.

A partir da modelagem e análise dessas séries temporais realizou-se um estudo preliminar envolvendo os diferentes métodos estatísticos com suavização exponencial para a previsão de venda e fabricação dos trimestres de 2016 de cada uma das duas categorias de produtos. Nesse estudo, avaliou-se a capacidade preditiva dos diferentes métodos com suavização exponencial por série, sob a premissa de conhecer os dados reais do ano de previsão. A tabela 1 mostra um resumo do desempenho relativo entre os diferentes métodos estatísticos de previsão por séries temporais com suavização exponencial avaliado para os dados desse trabalho, obtido através da medida estatística erro quadrático médio (MSE). 
Tabela 1 - Estudo comparativo do desempenho estatístico entre os métodos de previsão com suavização exponencial avaliados via erro quadrático médio (MSE)

\begin{tabular}{|c|c|c|c|c|c|}
\hline \multirow[b]{2}{*}{ Produto } & \multirow{2}{*}{$\begin{array}{l}\text { Método de } \\
\text { Previsão }\end{array}$} & \multicolumn{2}{|c|}{ Não Otimizado } & \multicolumn{2}{|r|}{ Otimizado } \\
\hline & & MSE & $\begin{array}{c}\text { Constante de } \\
\text { Suavização }\end{array}$ & MSE & $\begin{array}{c}\text { Constante de } \\
\text { Suavização }\end{array}$ \\
\hline E & $\begin{array}{l}\text { Suavização } \\
\text { Exponencial } \\
\text { (Simples) }\end{array}$ & 11,0 & $\alpha=0,5$ & 10,8 & $\alpha=0,397$ \\
\hline $\begin{array}{l}\mathrm{E} \\
\mathrm{T}\end{array}$ & Holt & 13,2 & $\alpha=0,5$ e $\beta=0,5$ & 9,0 & $\alpha=0,116$ e $\beta=1$ \\
\hline $\begin{array}{l}R \\
O \\
D\end{array}$ & $\begin{array}{l}\text { Holt-Winter } \\
\text { (Aditivo) }\end{array}$ & 14,8 & $\alpha=0,5 \quad \beta=0,5$ & 8,3 & $\begin{array}{c}\alpha=0,267 e^{\beta=0,105} \\
\gamma=1\end{array}$ \\
\hline $\begin{array}{l}\mathrm{U} \\
\mathrm{T} \\
\mathrm{O}\end{array}$ & $\begin{array}{l}\text { Holt-Winter } \\
\text { (Multiplicativo) }\end{array}$ & 13,3 & $\begin{array}{c}\alpha=0,5 e^{\beta=0,5} \\
\gamma=0,5\end{array}$ & 9,5 & $\begin{array}{c}\alpha=0,085 \text { e } \quad \beta=0 \\
\gamma=0,5\end{array}$ \\
\hline B & $\begin{array}{l}\text { Suavização } \\
\text { Exponencial } \\
\text { (Simples) }\end{array}$ & $2.960,5$ & $\alpha=0,5$ & $2.949,3$ & $\alpha=0,547$ \\
\hline$U$ & Holt & $3.406,3$ & $\alpha=0,5$ e $\beta=0,5$ & $2.788,3$ & $\alpha=0,393$ e $\beta=0,101$ \\
\hline C & $\begin{array}{l}\text { Holt-Winter } \\
\text { (Aditivo) }\end{array}$ & $4.768,6$ & $\begin{array}{c}\alpha=0,5 e^{\beta=0,5} \\
\gamma=0,5\end{array}$ & $3.337,6$ & $\begin{array}{c}\alpha=0,424 e^{\beta=0,075} \\
\gamma=1\end{array}$ \\
\hline$A$ & $\begin{array}{l}\text { Holt-Winter } \\
\text { (Multiplicativo) }\end{array}$ & $5.809,1$ & $\begin{array}{c}\alpha=0,5 \mathrm{e}^{\beta=0,5} \\
\gamma=0,5\end{array}$ & $3.810,1$ & $\begin{array}{c}\alpha=0,632 e^{\beta=0} \\
e^{\gamma=0,498}\end{array}$ \\
\hline
\end{tabular}

Fonte: Autores (2019)

Os resultados desse estudo preliminar, conforme tabela 1, mostram um melhor desempenho (valor ótimo da medida estatística MSE) do método com suavização exponencial dupla de Holt e do método de suavização exponencial tripla de HoltWinter (Aditivo) para as séries temporais dos produtos eletroduto e bucha, respectivamente. Assim, tais métodos são preferidos entre os demais métodos com suavização exponencial na previsão de valores futuros.

A sistemática de modelagem para estabelecer as previsões de demanda neste trabalho envolve a utilização de planilhas eletrônicas em ambiente MS-Exce|® com o objetivo de selecionar o método de previsão com suavização exponencial mais apropriado para as duas categorias de produto, ou seja, o método capaz de prever acontecimentos futuros, tendo como propósito a redução de riscos na tomada de decisão. Essa metodologia de aplicação para a previsão de demanda envolve a utilização de um algoritmo robusto que inclui basicamente um conjunto de cinco 
etapas que combinam todo o referencial teórico até aqui apresentado para estabelecer a previsão de demanda via ambiente MS-Excel® conforme a seguir.

1. Calcule o nível básico $E_{t}$, para o período de tempo t, utilizando a equação (3) para o método de Holt e a equação (7) para o método de Holt-Winter aditivo;

2. Calcule o valor da tendência estimada $T_{t}$, para o período de tempo t, utilizando equação (4) para o método de Holt e a equação (8) para o método de HoltWinter aditivo;

3. Calcule o fator Sazonal estimado $S_{t}$, para o período de tempo t, utilizando equação (9) para o método de Holt-Winter aditivo;

4. Calcule a previsão final $\hat{Y}_{t+n}$, para o período de tempo $t+n$, utilizando a equação (6) para os método multiplicativo e aditivo;

5. Resolva o modelo matemático programação não linear a seguir aplicando a ferramenta solver do ambiente MS-Excel® para determinar o modelo de previsão apropriado com a estimação dos parâmetros $\alpha, \beta$ e $\gamma$ (constantes de suavização) que minimizam a função objetivo MSE para determinar a previsão ótima, ou seja, resolver o modelo matemático de programação não linear a seguir.

\section{Minimizar MSE}

Alterando $\alpha, \beta$ e/ou $\gamma$

Sujeito a:

$0 \leq \alpha, \beta, \gamma \leq 1$

As previsões estabelecidas através dos dados reais pelos métodos com suavização exponencial são preliminarmente calculadas com os parâmetros (constantes de suavização) iguais a 0,5 para a série temporal dos produtos eletroduto e bucha, respectivamente. Em seguida, o modelo matemático de programação não linear é resolvido com a ferramenta solver do ambiente MS-Excel® para estimar os parâmetros, ou seja, as constantes de suavização que melhoram a previsão de demanda. As figuras 3 e 4 ilustram a solução ótima obtida com a estimação das constantes de suavização obtida com a ferramenta solver do ambiente MS-Exce|® que minimizam o MSE com objetivo de melhorar a previsão. 
Figura 3 - Solução ótima da previsão de demanda do produto eletroduto para o método com suavização exponencial tripla de Holt-Winter aditivo

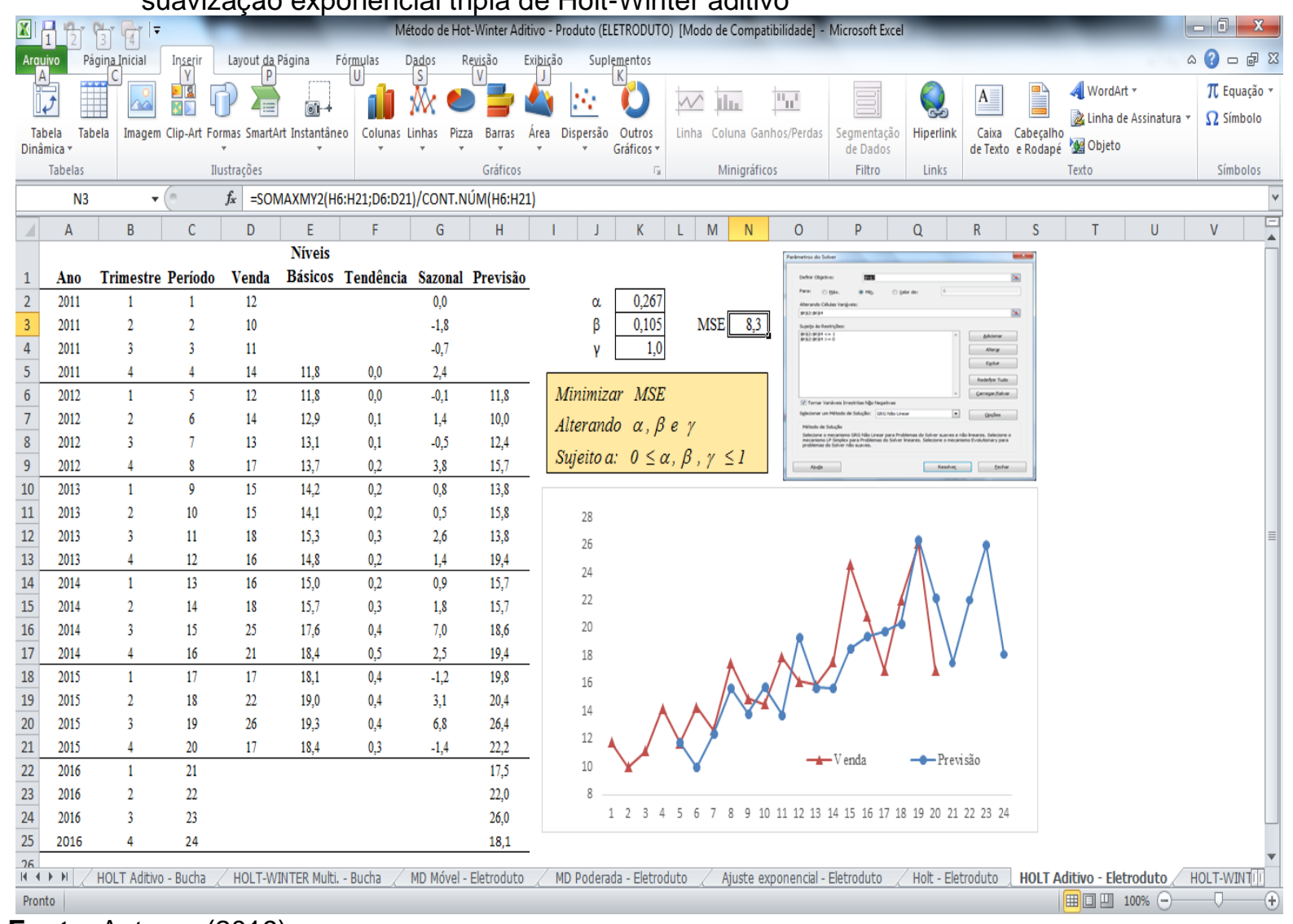

Fonte: Autores (2019)

\subsection{Resultados e discussão}

A análise estatística dos gráficos (figuras 3 e 4 ) revela que as alterações, ou seja, as minimizações das constantes de suavização produzem valores que tendem a aproximar-se dos dados originais. Assim, é possível obter-se um cenário de previsão que melhor reproduz as condições verificadas na série temporal. Além disso, comparando os resultados referentes ao valor do MSE entre os métodos com suavização estudados, o método com suavização exponencial tripla de Holt-Winter (Aditivo) e o método com suavização exponencial dupla de Holt são selecionados pelo fato de serem capazes de produzirem uma função que melhor se adapta aos dados da série dos produtos eletroduto e bucha, respectivamente.

Os resultados do desempenho estatístico relativo aos diferentes métodos com suavização exponencial obtidos neste estudo, a partir do valor do erro quadrático médio (MSE) conforme mostrado na tabela 1, confirmam o método estatístico com suavização exponencial tripla de Holt-Winter (Aditivo) conforme ilustrado na (figura 3) 
e o método estatístico com suavização exponencial dupla de Holt conforme ilustrado na (figura 4), como preferidos e selecionados por apresentarem melhor desempenho (grau de acurácia) em relação aos demais métodos de previsão com suavização exponencial estudados para estabelecer à previsão de demanda dos produtos eletroduto e bucha, respectivamente.

Figura 4 - Solução ótima da previsão de demanda do produto bucha para o método com suavização exponencial dupla de Holt

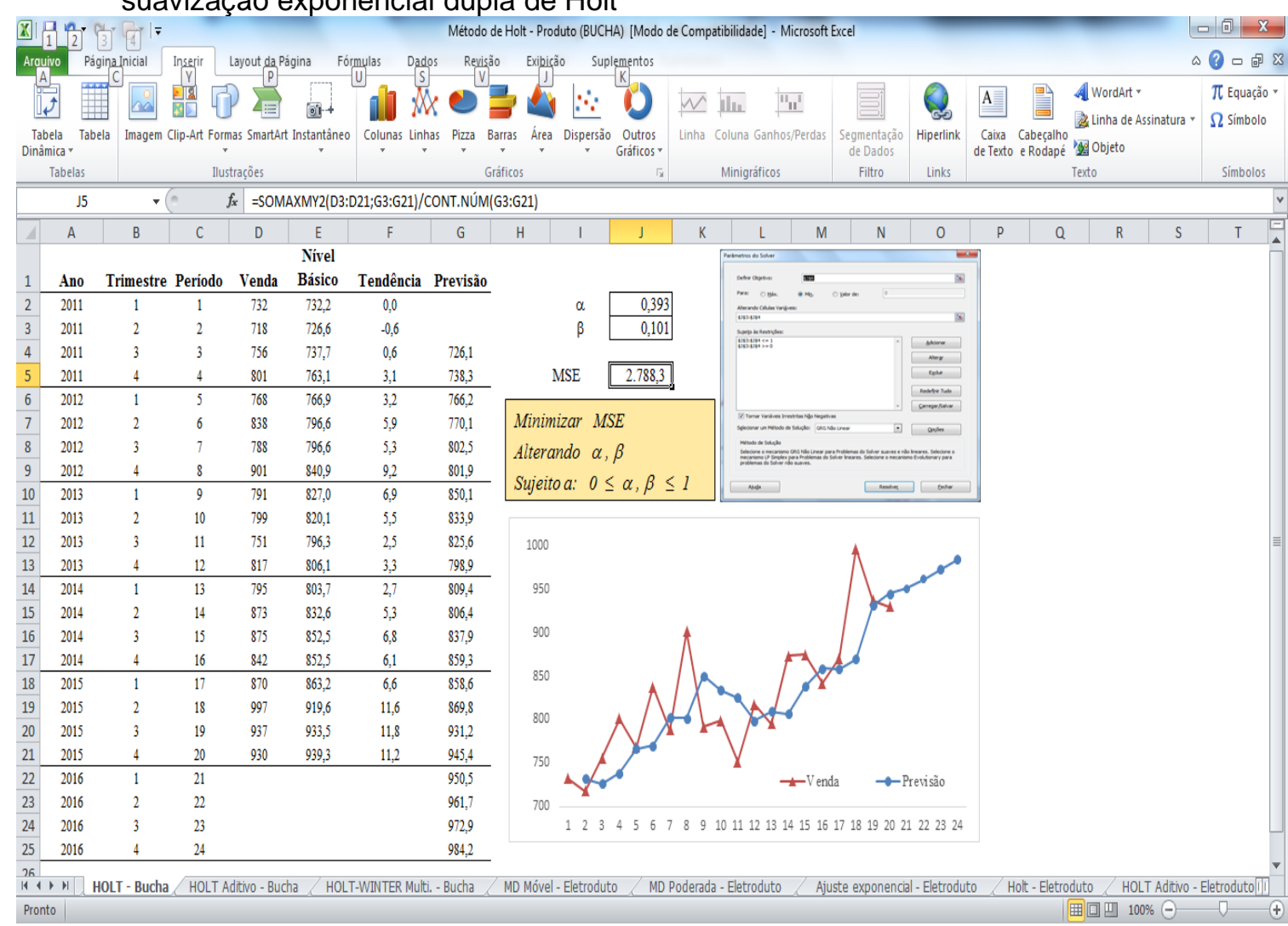

Fonte: Autores (2019)

Os resultados obtidos com a seleção apropriada desses métodos preditivos com suavização exponencial, objeto de estudo deste trabalho, foram fundamentais para o analista do sistema de previsão de demanda direcionar especial atenção ao grau de acurácia que integrado a uma política de gerenciamento de estoques eficiente foi responsável por minimizar os efeitos de variabilidade e custos operacionais, além contribuir na melhoria dos níveis de serviço e no consequente aumento da rentabilidade da indústria envolvida. 


\section{CONSIDERAÇÕES FINAIS}

As empresas atualmente estão enfrentando uma nova economia globalizada motivada por desafios cada vez mais exigentes. Hoje, seus concorrentes podem estar em qualquer lugar do planeta. Este contexto de ambiente globalizado com extrema concorrência tem despertado cada vez mais a indústria moderna a adotar políticas que possam assegurar a sua sobrevivência e competitividade, através da tomada de decisões de suas atividades de gestão com o propósito de atingir níveis satisfatórios de eficiência nas suas operações e processos gerenciais, bem como a redução de custos.

O objetivo principal da aplicação de métodos estatísticos com suavização exponencial propostos neste trabalho para estabelecer a previsão de demanda integrado às políticas de gestão de estoques com o propósito de antecipar cenários futuros de vendas para as duas categorias de produtos foi atingido cujos resultados obtidos sinalizaram o melhor aproveitamento da capacidade produtiva através de uma gestão de estoques adequada para a redução de riscos no processo de tomada de decisões gerenciais dessa indústria. Tais resultados foram fundamentais para o analista do sistema de previsão de demanda direcionar especial atenção ao grau de acurácia que integrado a uma política de gerenciamento de estoques eficiente foi responsável por minimizar os efeitos de variabilidade e custos operacionais, além contribuir na melhoria dos níveis de serviço e no consequente aumento da rentabilidade da indústria envolvida. Esses resultados foram considerados satisfatórios na medida em que cumpriram os objetivos inicialmente propostos e favoreceram o compromisso de melhoria contínua no monitoramento processo logístico da indústria envolvida.

Não foi possível quantificar neste estudo os valores monetários (em $R \$$ ) concretos seja nos produtos e/ou seja no monitoramento do processo logístico, fonte de custos importantes que engloba as principais atividades de apoio à logística ligadas à posse e movimentação dos produtos como previsão de demanda, gestão de estoques, transportes, armazenagem, etc. No entanto, partindo de indicadores como a produtividade, redução de estoques e avarias, pode concluir-se que todos esses indicadores denotam melhorias que, seguramente, foram decisivas na redução de custos. Além disso, sabe-se ainda que a efetiva aplicação da metodologia proposta 
via métodos estatísticos com suavização exponencial dupla e tripla para estabelecer a previsão de demanda através de dados reais referentes ao histórico de vendas da empresa poderá refletir-se, a longo prazo, numa maior e melhor organização da gestão da produção e estoques com resultados bastante positivos para 0 monitoramento logístico no que diz respeito aos custos.

A seleção adequada do método estatístico com suavização exponencial potencialmente capaz de estabelecer a melhor previsão de demanda dos produtos (eletroduto e bucha), apresentaram vantagens que contribuíram para o aumento significativo do grau de acurácia da previsão, considerando-se aspectos estatísticos e econômicos. Sob o ponto de vista estatístico, a redução do valor do MSE com a otimização dos parâmetros estatísticos de suavização exponencial (constantes de suavização: $\alpha, \beta$ e/ou $\gamma$ ) do método de previsão via ferramenta Solver do MS-Excel®. Sob o ponto de vista econômico, a acurácia da previsão de ambos os produtos proporcionou uma redução de custos relacionados principalmente a pagamentos extras decorrentes de alterações de programações de produção. Obviamente, a redução de custos obtida com a seleção apropriada dos métodos estatísticos responsáveis pela otimização da previsão de demanda (mínimo valor de MSE), pode ser bastante significativa, principalmente porque envolvem desvios da meta de produção estabelecida desses produtos que implicam em outros prejuízos materiais.

É importante salientar ainda que a adoção de qualquer método estatístico de previsão pressupõe uma escolha adequada deste e de seus parâmetros, uma vez que o grau de acurácia da previsão de demanda depende em parte destas escolhas.

\section{REFERÊNCIAS}

BERMÚDEZ, J.D.; SEGURA, J.V.; VERCHER, E. SIOPRED: a prediction and optimization integrated system for demand. TOP, v.16, n.2, p. 258-271, feb. 2008.

https://doi.org/10.1007/s11750-008-0042-7

BILLAH, B.; KING, M.L.; SNYDER, R.D.; KOEHLER, A.B. Exponential smoothing model selection for forecasting. International Journal of Forecasting, v.22, n.2, p. 239-247, jun.2006. https://doi.org/10.1016/j.ijforecast.2005.08.002

BOWERSOX, D.J.; CLOSS, D.J.; COOPER, M.B.; BOWERSOX, J.C. Gestão logística da cadeia de suprimentos. 4. ed. Porto Alegre: AMGH, 2014.

BROWN, R.G. Statistical forecasting for inventory control. New York: McGraw-Hill, 1959. 
COELHO, L. C. Utilização de modelos de suavização exponencial para previsão de demanda com gráficos de controle combinados Shewhart-CUSUM. 121p. Dissertação (Mestrado) - Universidade Federal de Santa Catarina, Florianópolis, 2008.

COELHO, L. C. Flexibility and Consistency in Inventory-Routing. 183 p.

Canadá: Thèse (Doctorat) - Hec Montréal, Affiliée à L'Université de Montréal, 2012.

COELHO, L.C. Gestão e previsão da demanda. In: CALLEGARI COELHO, Leandro. Logística Descomplicada: demanda, gestão, logística, previsão. Québec, Canadá: Université Laval, Disponível em: https://www.logisticadescomplicada.com/gestao-previsaodemanda/. Acesso em: $10 \mathrm{dez} .2018$.

COELHO, L.C.; LAPORT, G. The exact solution of several classes of inventory-routing problems. Computers \& Operations Research, v.40, n.2, p.558-565,

feb. 2013. https://doi.org/10.1016/j.cor.2012.08.012

COUTO \& ESPÍRITO SANTO, A gestão de estoques baseada na previsão de demanda: um estudo em um jogo de empresas. Revista LAGOS - UFF, Volta

Redonda, v. 4, n.2, p.83-96, Abr.2013. https://doi.org/10.1002/ad.1562

Disponível em: http://www.revistalagos.uff.br/index.php/lagos/article/view/231

CRUZ, A.C., AMARAL, C.E.; ALVES, C. C., HENNING, E.; CORAZZA, E.J. Avaliação e Monitoramento do Desempenho de Modelos de Previsão, IV Congresso Brasileiro de Engenharia de Produção, Anais COMBREPRO 2014, Ponta Grossa: APREPRO, 2014. Disponível em: http://www.aprepro.org.br/conbrepro/2014/anais/artigos/eng\%20q/2.pdf Acesso em: 18 de dezembro de 2018.

GARCÍA-DÍAZ, J.C. Predición en el dominio del tiempo: analisis de series temporales para ingenieros.1. ed. España: Editorial Universitat Politècnica de València, 2016.

GARDNER E.S. Jr. Exponential Smoothing: The State of the Art. Journal of Forecasting, v.4, p.1-28, 1985. https://doi.org/10.1002/for.3980040103

Disponível em: https://www.bauer.uh.edu/gardner/docs/pdf/Exp-Sm-1985.pdf

GARDNER E.S. 2006. Exponential Smoothing: The State of the Art - Part II. International Journal of Forecasting, v.22, n.4, p. 637-666, 2006.

https://doi.org/10.1016/j.ijforecast.2006.03.005

GELPER, S.; FRIED, R.; CROUX, C. Robust forecasting with exponential and Holt-Winters smoothing. Journal of Forecasting, v.29, 285-300, 2010. https://doi.org/10.1002/for.1125 Disponível em: https://pure.tue.nl/ws/portalfiles/portal/3954158/671965904247136.pdf

HEIZER, J.H, RENDER, B. and MUNSON, C. Operations management: sustainability and supply chain management, 12th edition, Boston Pearson, 2017.

HYNDMAN, R.J. \& ATHANASOPOULOS, G. Forecasting: principles and practice, otexts. Disponível em: https://www.otexts.org/book/fpp. Austrália: University of Western Australia, 2018.

HOLT, C.E. Forecasting trends and seasonal by exponentially weighted averages. ONR Memorandum, v.52, Carnegie Institute of Technology, Pittsburgh, USA,1957. 
JUÁREZ, A.C.; ZUÑIGA,C.A.; FLORES, L.M.; PARTIDA, D.S. Análisis de series de tiempo en el pronóstico de la demanda de almacenamiento de productos perecederos. Estudios Gerenciales, 32, 387-396, 2016. https://doi.org/10.1016/j.estger.2016.11.002

Disponível em: https://www.redalyc.org/articulo.oa?id=21251783011

KRAJEWSKI, L.J.; MALHOTRA, M.K.; RITZMAN, L.P. Operations Management: processes and supply chains plus, 11th Edition, Prentice Hall, Upper Saddle River, 2015.

LÓPEZ, J.A.; MENDOZA, A.; MASINI, J. A Classic and effective approach to inventory management, International Journal of Industrial Engineering, v. 20, n. 5/6, p.372-386, 2013.

MAKRIDAKIS, S.; WHEELWRIGHT; S.; HYNDMAN, R.J. Forecasting methods and applications. 3rd ed., Wiley India Pvt. Limited, 2008.

MONTGOMERY, D.C.; JENNINGS, C.L.; KULAHCI, M. 2016. Introduction to time series analysis and forecasting, Edition $2^{\text {th }}$, John Wiley \& Sons, 2016.

PELLEGRINI, F.R. \& FOLIATTO, F.S. Passos para Implantação de Sistemas de Previsão de Demanda -Técnicas e Estudo de Caso. Revista Produção, v.11, n.1 p.43-64, nov. 2001. https://doi.org/10.1590/S0103-65132001000100004 Disponível em: Disponível em:

http://www.scielo.br/pdf/prod/v11n1/v11n1a04

PINEDA, D.E.S. and TORRES, N.R. Inventory management model design in a strawberry crop, based on the model order for a single period and six sigma metrics, Ingeniería y Competitividad, vol.1, n.1, p.95-105, 2018. Disponível em:

Disponível em: http://www.scielo.org.co/pdf/inco/v20n1/0123-3033-inco-20-01-00095.pdf

MORETTIN, P. A. \& TOLOI, C. M. Análise de séries temporais. 2. ed., Editora Edgard Blucher, 2006.

RAGSDALE, C. Spreadsheet modeling and decision analysis: a practical introduction to business analytics. Cengage Learning, 8th edition, 2017.

RASMUSSEN, R. On time series data and optimal parameters. Omega, 32, 111-120, 2004. https://doi.org/10.1016/j.omega.2003.09.013. Disponível em:

http://citeseerx.ist.psu.edu/viewdoc/download?doi=10.1.1.530.8034\&rep=rep1\&type=pdf

SINGH, H., BAHL, A. \& KUMAR, A. Application of time-series demand forecasting models with seasonality and trend components for industrial products. International Journal of Mechanical Engineering and Technology (IJMET), v.8, n.7, p.1599-1606, 2017. Disponível em: https://www.iaeme.com/MasterAdmin/uploadfolder/IJMET $08 \quad 07$ 176/IJMET 0807 176.pd f

SOUZA, G.P.; SAMOHYL, R.W. \& MIRANDA, R.G. Métodos simplificados de previsão empresarial. Editora Ciência Moderna, 2008.

VARGAS, E.J.; BACCIN, B.; SELLITTO, M.A. Análise integrada para a tomada de decisão: os efeitos da modelagem no gerenciamento de estoques e o impacto sobre o indicador de rentabilidade ROI. Revista Produção Online, v.17, n.1, p. 325-350, jan./mar., 2017. https://doi.org/10.14488/1676-1901.v17i1.2556. Disponível em: https://producaoonline.org.br/rpo/article/view/2556 
VERíSSIMO, A. J., ALVES, C. C., HENNING, E., AMARAL, C.E. \& CRUZ, A.C. Métodos Estatísticos de Suavização Exponencial Holt-Winters para Previsão de Demanda em uma Empresa do Setor Metal Mecânico - Gestão Industrial, 8, p.154-171, 2012. https://doi.org/10.3895/S1808-04482012000400009. Disponível em: https://periodicos.utfpr.edu.br/revistagi/article/view/1378/922

WINTERS, P. R. Forecasting sales by exponentially weighted moving averages Management Science, v.6, n.3, p.324-342, 1960. https://doi.org/10.1287/mnsc.6.3.324

YORK, J.C. Evaluating the performance and accuracy of forecasting incident rates for mining operations. 189 p. Dissertation (Master), Pennsylvania State University, Graduate School College of Earth and Mineral Sciences, 2015.

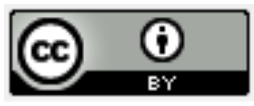

Artigo recebido em:12/01/2019 e aceito para publicação em: 30/08/2019

DOI: http://dx.doi.org/10.14488/1676-1901.v19i3.3539539 